\title{
Recomendaciones generales en Periodoncia en momento de pandemia
}

Prof. Dr. Luis Bueno

Cátedra de Periodoncia. Facultad de Odontología. UdelaR

ORCID: 0000-0002-8442-3005

Introducción

En este momento tan difícil de la pandemia se indica, bajo las condiciones indicadas en esta guía, consulta telefónica con el paciente para evacuar la urgencia y en su defecto proceder atender la misma, si es que telefónicamente es imposible solucionarla.

Para esto, a continuación se describen los cuadros gingivoperiodontales que merecen atención de urgencia en periodoncia, cuadros que se caracterizan por su inicio rápido que involucran el periodonto o las estructuras asociadas y pueden caracterizarse por dolor así como por destrucción de los tejidos e infección. (1)

En el caso que el síntoma sea dolor y el profesional considere pertinente, se recomienda indicar correcta medicación analgésica, de ser oportuno el tratamiento antibiótico, según el caso (como se describe a continuación) y otras instrucciones o conductas a seguir, con el correcto monitoreo por parte del profesional vía telefónica.

En el caso de ser impostergable la atención de manera presencial, se hace énfasis en respetar todas las medidas de bioseguridad mencionadas en esta guía.

Cuadros Periodontales que merecen atención de urgencia

\section{1- Absceso periodontal}

El absceso periodontal es una infección purulenta localizada en los tejidos periodontales, comúnmente encontrado en pacientes con enfermedad periodontal. Suele estar asociado a bolsas periodontales profundas, de recorrido tortuoso y en zonas de furcación. Es importante para su diagnóstico la evaluación del paciente, interpretación de su sintomatología, historia médica y odontológica (indagar antecedentes de tratamiento periodontal) y el examen radiográfico (de tenerlo disponible enviar fotos de las radiografías).

El tratamiento sintomático convencional de los abscesos periodontales incluye drenaje y desbridamiento, frecuentemente en combinación con tratamiento con antibióticos sistémicos. 
Como primer medida en este momento tan especial se sugiere antibioterapia, las drogas sugeridas son las siguientes:

- Metronidazol (comúnmente prescrito para condiciones agudas 250mg c/8 horas. En Uruguay esta disponible en dosis de $500 \mathrm{mg}$ que debe ser administrado c/8 hrs)

- Amoxicilina con ácido clavulánico (875 + 125 mg, c/12 horas disponible en Uruguay).

- Azitromicina (500 mg, c/24 horas, 5 días, disponible en Uruguay ${ }^{(2)}$.

Varios autores recomiendan extender el tratamiento antibiótico mientras dure la etapa aguda, que suele resolverse en menos de 5 días. ${ }^{(3,4)}$

La Cátedra de Periodoncia recomienda completar el uso del antibiótico de 7 días ( Metronidazol o Amoxicilina siendo el Metronidazol la droga de primera elección en estos cuadros).

Es importante tener en cuenta que, ninguno de estos regímenes de antibióticos van a resolver enteramente la causa del absceso, lo cual implica que el tratamiento de su etiología deberá ser realizado en el momento apropiado. ${ }^{(5)}$

Una vez cumplida la semana de antibioterapia se evalúa telefónicamente la evolución del cuadro.

Se recuerda al paciente el refuerzo de las medidas de higiene mecánica dental y lingual así como la coadyuvancia antiséptica (idealmente clorhexidina $0,12 \%$ dos veces al día).

\section{2- Enfermedades periodontales necrotizantes}

Son un grupo de enfermedades infecciosas que incluyen a la Gingivitis Ulceronecrotizante, Periodontitis Ulceronecrotizante y Estomatitis Necrotizante. Un síntoma característico es dolor y un factor de riesgo es el stress (tan presente en este momento).

Los hallazgos clínicos más relevantes de la Gingivitis Necrotizante incluyen: dolor, necrosis y úlcera de la papila interdental, sangrado gingival, formación de un esfacelo blancoamarillento, halitosis, linfoadenopatías y fiebre. La Periodontitis Necrotizante presenta, además de los signos anteriores, pérdida de inserción periodontal y destrucción ósea. En el caso de la Estomatitis Necrotizante, la denudación ósea se extiende a través de la mucosa alveolar. ${ }^{(6)}$

Para el tratamiento de la fase aguda, se indica Metronidazol 500mg/8 horas 7 días. (7)

Para el tratamiento en el consultorio se recomienda un desbridamiento cuidadoso del biofilm

y tártaro presente. Por lo general, son de elección los instrumentos ultrasónicos porque 
generan menos molestia para el paciente, pero de ser posible en estas circunstancias por el alto riesgo al generar aerosoles se sugiere utilizar instrumentos manuales, lo cuales son eficaces. ${ }^{(8)}$

La literatura es muy clara en que la aerosolización de secreciones, saliva o sangre puede aumentar con el uso de instrumental ultrasónico, con el riesgo de contraer la enfermedad virósica. ${ }^{(9-10)}$

En caso de tartar la urgencia de forma presencial el desbridamiento debería de ser realizado diariamente y a mayor profundidad a medida que el paciente lo tolere, mientras que dure la fase aguda (generalmente de 2 a 4 días). Indicar al paciente evitar el control mecánico del biofilm, ya que el mismo puede impedir la cicatrización de las úlceras. El control químico del biofilm está indicado, con clorhexidina $(0.12 \%$, cada 12 horas) o peróxido de hidrógeno $3 \%$ diluído 1:1 en agua tibia. Son cuadros que deben ser acompañados siempre con analgésicos menores. ${ }^{(11)}$

\section{3- Gingivoestomatitis Herpética Aguda}

Es causada por la infección del HSV-1 y se caracteriza por la formación de vesículas que se rompen, se unen y dejan úlceras recubiertas de fibrina a menudo de extensión irregular. Es otro cuadro que el síntoma a destacar es dolor.

Es importante realizar un diagnóstico diferencial con otras condiciones como estomatitis aftosa, ya que las últimas lesiones no afectan el tejido queratinizado. ${ }^{(12)}$ La condición es autolimitante, el tratamiento incluye dieta líquida o blanda, correcta hidratación (componente fundamental), remoción atraumática de biofilm, enjuagatorios de clorhexidina al 0,12\%, para limitar la superinfección de las úlceras, lo cual retrasa su cicatrización. En casos severos y pacientes con inmunodeficiencia, el uso sistémico de drogas antivirales como Aciclovir, Valaciclovir está recomendado. ${ }^{(13)}$ Se sugiere la indicación de analgésicos y enjuagues orales para que el paciente se sienta mejor y facilite la ingesta de líquidos dando lugar a una correcta hidratación. (14)

La consulta telefónica con una anamnesis correcta acompañada por fotos enviadas por el paciente puede permitir llegar al diagnóstico correcto y eventual guía de tratamiento.

\section{Antisépticos en Periodoncia Y COVID 19}

La saliva y el colutorio juegan factores importantes en los tratamientos odontológicos. El SARS-CoV-2 puede estar presente en saliva mediante tres mecanismos principales: 
- Intercambio de fluídos y gotículas provenientes de las vías aéreas superiores e inferiores (Gotitas de Flugge)

- Suero sanguíneo que se libera al líquido crevicular gingival

- Infección en las glándulas salivales, con subsecuente descarga de partículas virales en la saliva. (15)

Durante los 10 primeros días, en los que el paciente es asintomático y altamente contagioso, la infección se localiza en vías respiratorias superiores (boca, nariz, garganta). Posteriormente, con la evolución de la enfermedad, el virus pasa a vías respiratorias bajas (pulmones) (16). En humanos se ha aislado ARN viral en saliva en la fase inicial de la enfermedad (17).

Los tratamientos dentales invariablemente conllevan el riesgo de infección con el virus SARS-CoV-2 debido a la especificidad de sus procedimientos que implica la cercanía con el paciente, la consecuente exposición a la saliva, sangre, fluídos y el uso de instrumental odontológico rotatorio, por lo que es necesario el control y disminución de microorganismos que existen en la cavidad oral.

El coronavirus SARS-CoV infecta a las células a través de receptores ECA2, estos receptores se encuentran en alta densidad especialmente en la lengua ${ }^{(18)}$. La carga viral está altamente relacionado con la gravedad de COVID-19, siendo la cavidad bucal un foco de alto riesgo de infección. ${ }^{(19)}$ La disminución de la carga patógena en la cavidad bucal ayudaría a reducir la gravedad de la enfermedad COVID-19 y a reducir temporalmente la transmisión. En general, un enjuague bucal antimicrobiano preoperatorio reduce la cantidad de microbiota oral. Sin embargo, según las instrucciones de la "Guideline for the Diagnosis and Treatment of Novel Coronavirus Pneumonia (the 5th edition)" la clorhexidina, que se usa comúnmente como antiséptico en la práctica dental, no se recomienda contra Covid -19 . Siendo el virus vulnerable a la oxidación, son eficaces los enjuagues bucales que contienen agentes oxidativos tales como Peróxido de Hidrógeno al $1 \%$ o 0, 2\% de Povidona odada. Por tales motivos se recomienda el enjuague bucal previo a los procedimientos del tratamiento odontológico. ${ }^{(20)}$

Otro de los compuestos sugeridos actualmente, es el Cloruro de Cetilpiridinio (CPC).

Estudios in vitro e in vivo muestran actividad virucida y preventiva del CPC frente a algunos virus, incluyendo algunas cepas de coronavirus. EI CPC altera la membrana lipídica de virus con envuelta lipídica a través de interacciones fisicoquímicas, provocan su ruptúra y, por tanto, la inactivación del mismo. No generando viriones resistentes al CPC. (21)

En un un ensayo clínico realizado en 94 voluntarios sanos con el objetivo de saber si el uso de CPC aplicado oralmente podría prevenir infecciones de vías respiratorias superiores 
causadas por virus de la influenza, el virus respiratorio sincicial, metapneumovirus humano, rhinovirus y adenovirus se concluyó que el CPC es eficaz en la prevención de infecciones de vías respiratorias superiores por virus con envuelta lipídica. El CPC tiene una sustantividad de 3 a 5 horas, debe realizarse enjuagues de $20 \mathrm{ml}$ durante 1 minuto, 3 veces al día, en este caso evitando salivar, aspirando con el suctor. ${ }^{(22)}$

\section{Recomendaciones acerca de la Higiene Bucodental}

El concepto general de las visitas de mantenimiento posterior a los tratamientos periodontales tiene por objetivo minimizar la recurrencia de la enfermedad a través de intervenciones periódicas. Una visita de mantenimiento se considera una etapa obligatoria de tratamiento en los pacientes con patologia periodontal ya que se trata de una afección crónica. ${ }^{(23)}$

En este contexto de pandemia las terapias de mantenimiento de los pacientes periodontales se posponen quedando la salud periodontal unicamente dependiente del comportamiento del paciente en relación a la higiene bucal.

Los cepillos de dientes, que deben ser siempre de cerdas suaves, se contaminan con los microorganismos presentes en la cavidad bucal. La retención y la supervivencia de microorganismos en este después del cepillado representa una posible causa de contaminación de la boca. ${ }^{(24)}$

Numerosos estudios han demostrado que el uso prolongado del cepillo de dientes facilita la contaminación por diversos microorganismos. ${ }^{(25)}$

Recomendamos, en lo posible, el uso de capuchón con ventilación (protección de contaminación), guardarlo en posición vertical, separados unos de otros (utilizando un vaso para cada cepillo) y cambiar el cepillo tras un proceso infeccioso.

Frente a la presencia de alta densidad de receptores ECA2 (receptores de afinidad de SARSCoV-2) en la lengua.1 indica el uso de limpiador lingual, el cual ha demostrado una reducción del cubrimiento lingual, células epiteliales y microorganismos.

La desinfección deL cepillo dental es de gran importancia para mantener su correcta limpieza. También es recomendable que no se comparta el mismo tubo de pasta dental, ya que puede favorecer la contaminación cruzada. 


\section{Recomendaciones}

1- Evite uso de cavitador por generacion de aerosoles

2- Escuche atentamente al paciente durante la consulta telefónica e indague sobre toda su sintomatologia. Vea la posibilidad de que se envíe foto de la zona y posibles estudios radiográficos si los tiene (en caso de indicar, se prefiere ortopantomografía) evitando la consulta presencial, si es posible.

3- La indicacion de analgesia y antibióticos en casos de urgencias vía telefónica puede, en muchos casos, permitir dilatar la consulta presencial.

4- Para campo interno en caso de tener que tratar una urgencia no se indica clorhexidina $0,12 \%$ ya que no es efectiva para Covid-19.

5- Para el tratamiento domiciliario de un posible cuadro periodontal de urgencia que necesite coadyuvancia antiséptica indicamos clorhexidina $0,12 \%$ para el cuadro periodontal específico.

\section{Referencias}

1- American Academy of Periodontology. Parameter On Acute Periodontal Diseases. J Periodontol 2000; 71(5-s): 863-866.

2- Herrera D, Alonso B, De Arriba L, Santa Cruz I., Serrano C., Sanz M. Acute periodontal lesions. Periodontal 2000 2014; 65:149-177.

3 Herrera, D; Winkelhoff, V; ; Sanz, M. Abscesses in the periodontium and Implant Dentistry. 6ed. Oxford: Blackwell Munksgaard, 2015, p- 463-469.

4- Holmstrup, P. Necrotizing periodontal disease. En: Lang, N; Lindhe, J. Clinical Periodontology and Implant Dentistry. 6ed. Oxford: Blackwell Munksgaard, 2015. P- 421-434.

5- Herrera D, Retamal $\square$ Valdes B, Alonso B, Feres M. Acute periodontal lesions (periodontal abscesses and necrotizing periodontal diseases) and endo $\square$ periodontal lesions. J Clin Periodontol. 2018;45(Suppl 20):S78-S94.

6- Loesche WJ, Syed SA, Laughon BE, Stoll J. The bacteriology of acute necrotizing ulcerative gingivitis. J Periodontol 1982: 53: 223-230.

7- Suvan, J, Leira Y, Moreno F, Graziani F, Derks J, Tomasi C. Subgingival Instrumentation for Treatment of Periodontitis. A Systematic Review. J Clin Periodontol. 2019 Dec 30. doi: 10.1111/jcpe.13245

8- L. Meng, F. Hua , Z. Bian. Coronavirus Disease 2019 (COVID-19): Emerging and Future Challenges for Dental and Oral Medicine. J Dental Res 2020: 99(5): 481-487. 
9- Harrel SK., Molinari J. Aerosols and splatter in dentistry A brief review of the literature and infection control implications J Am Dent Assoc 2004;135 (4), April: 429- 437.

10 - Wennstrom J, Lindhe J. Effect of hydrogen peroxide on developing plaque and gingivitis in man. J Clin Periodontol 1979: 6: 115-130.

11 - Scully C, Gorsky M, Lozada-Nur F. The diagnosis and management of recurrent aphthous stomatitis: a consensus approach. J Am Dent Assoc 2003: 134: 200-207.

12- O’Brien JJ, Campoli $\square$ Richards DM. Acyclovir. An update of its role in antiviral therapy. Current Therapeutics 1989: 30: 81-93.

13- Aslanova M, Zito PM. Herpetic Gingivostomatitis. [Updated 2020 Jan 7]. In: StatPearls [Internet]. Treasure Island (FL): StatPearl, 2020. Disponible en:

\section{https://www.ncbi.nlm.nih.gov/books/NBK526068/}

14- Eder A, Sigua J, Baernal A. COVID-19 y la Odontología: una Revisión de las recomendaciones y perspectivas para Latinoamérica. Int. J. Odontostomat 2020; 14(3): 299309.

15- Wölfel R; Corman; V; Guggemos W. Virological assessment of hospitalized patients with COVID-2019. Nature 2020. Disponible en: https://doi.org/10.1038/s41586-020-2196-X 16- Wang WK., Chen SY, Liu IJ, Chen YC, Chen HL, Yang CF, Chen PJ, Yeh SH., Kao CL., Huang LM. Detection of SARS-associated coronavirus in throat wash andsaliva in early diagnosis. Emerg. Infect. Dis 2004; 10(7):1213-9.

17- Xu H, Zhong L, Deng J, Peng J, Dan H, Zeng X, et al.High expression of ACE2 receptor of 2019-nCoV on the epithelial cells of oral mucosa. Int J Oral Sci. 2020 Feb 24;12(1):8.

18- Liu Y, Yan LM, Wan L, Xiang TX, Le A, Liu JM, Peiris M, Poon LLM, Zhang W. Viral dynamics in mild and severe cases of COVID-19. Lancet Infect Dis. 2020 Mar 19. Disponible en: https://www.ncbi.nlm.nih.gov/pmc/articles/PMC7158902/

20- Peng X, Xu X, Li Y, Chen L, Zhou X, Ren B, Transmission routes of 2019-nCov and controls in dental practice, Int J Oral Sci 2020; 12(1). Disponible en:

https://www.nature.com/articles/s41368-020-0075-9

Acceso 5/4/2020

21- Popkin DL, Zilka S, Dimaano M, Fujioka H, Rackley C, Salata R et al. Cetylpyridinium chloride (CPC) exhibits potent, rapid activity gainst influenza viruses in vitro and in vivo. Pathogens Immunity 2017; 2(2):253-69.

22- Mukherjee PK, Esper F, Buchheit K, Arters K, Adkins I, Ghannoum MA et al. Randomized, double-blind, placebo-controlled clinical trial to assess the safety and effectiveness of a novel dual-action oral topical formulation against upper respiratory infections. BMC Infect Dis. 2017 Jan $14 ; 17(1): 2-8$. 
23- Armitage, G; Xenoudi, P. Post-treatment supportive care for the natural dentition and dental implants Periodontology 2000 2016; 71: 164-184.

24-Wetzel W, Schaumburg C, Ansari F. Microbial contamination of toothbrushes with different principles of filament anchoring. J Am Dent Assoc. 2005; 136: 758-65.

25- Sogi S, Subbareddy V, Kiran S. Contamination of tooth brushes at different time intervals and effectiveness of various disinfection solutions in reducing the contamination of tooth brush. J Indian Soc Ped Prev Dent 2002; 20:81-5.

26-Karibasappa G, Nagesh L, Sujatha B. Assessment of microbial contamination of toothbrush head: An in vitro study. Indian J Dent Res. 2011; 22(1): 2-5.

27- Mukherjee P, Esper F, Buchheit K. Randomized, double-blind, placebo-controlled clinical trial to assess the safety and effectiveness of a novel dual-action oral topical formulation against upper respiratory infections. BMC Infect Dis. 2017 Jan 14; 17(1):2-8. 